Historic, Archive Document

Do not assume content reflects current scientific knowledge, policies, or practices. 
-Garden virseries, Narberth, Pe.

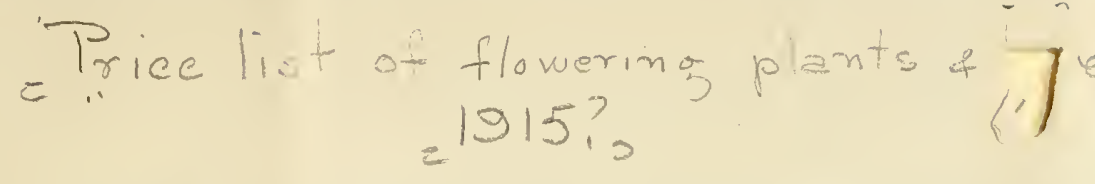

Triliium - Grouna Iily.

T. Erectum. Purple hrown; 1 foot; 5 th inonth.

T. Grandiflorum. "hite; 1 to lis fiot; 5 th month.

Tricyrtis Hirta - Japencse Toad Iily; White; 1 to 3 feet; 8-9 month.

Tritoma - Torch Iily.

T. Pfitzerii. Orango Scarlet; 3 -4 feet; 8-loth month.

T. Rufa. Cenary yellow, turning to orango-red as the flower ages; $2 \mathrm{ft}$.

6-11th month. Price, $25 \%$ each; 2.50 per dozen; \$15.00 per hundred.

Trollius - Globo Flower.

T. Asiaticus flore croceo. Orange; 30 inches; $5-6$ th month.

T. Caucasicus "Orange Globe". 2 feet; 5-6th month.

T. Iuropaeus. Iemon Yellov; 15 inches to 2 feet; 5 -8th month.

T. Fire Globe. Glowing Orange. 2 feet; 5 -7th month.

Voronica - Speedwell.

V. Amethystina. Blue; 1 to 3 feet; 5 -6th month.

V. Ionfifolia Subsessilis. Blue; 2 feet; 8-loth month.

V. Virginica. White or pale blue; $2-5$ feet; 8-9th month.

Vinca Minor - Periwinkle.

Dark blue, three to four inches.

Strons, transplanted nursory grom plants

Strone plants astablistec in 4 inch pots,

Strong collectec plants, 8 inches,

cach doz. 100

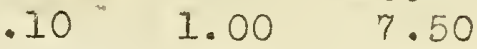

$\begin{array}{lll}.15 & 1.50 \quad 10.00\end{array}$

Viola Cornuta - Tuftec Pansies,

$\begin{array}{lll}.75 & 5.00 & 45.00\end{array}$

Viola cornuta - Tufec Pansies,

$.10 \quad 1.00 \quad 7.50$

Yucca Filamentosa - Aam's Noedie. Creamy-

white flowers. Tro year old plants

specimen plants

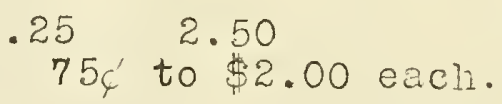

A collection of Evorgreens at a Spccial Price of $\$ 15.00$

1 Koster's Blue Spruce, imported, $2 \frac{1}{2}$ feet high.

I Retinospora Plumosa, Japan plune cedar, green, 24 inches.

1 Retinospora Filifera. Japan thread-like Codar, green, 30 inches.

I Retinospora Plumosa Aurea. Golcien plume cedar, 30 inchos.

2 Thuya Occidentalis. American Arbor Vitee; 3 feet.

1 Euonimus Japonica, grecn Elossy leaves.

1 Thuya Compacta. Globe Arbor Vitne, green.

2 Borwood Bushes. Green, 18 inches.

I Biota Aurea Nana. Golden Chinese Cedar

I Euonimus Vegetus. Groen foliage, red fruit in winter.

Twelve choice evergreens, enough for a small bed, for \$15.00 delivered.

Choice PAISIES, strong plants, finest varieties, $65 \varnothing$ per dozen; $\$ 5.00$ per hundred. Whon personally selected by customer direct from our frames, $75 \%$ per dozen; $\$ 5.50$ per hundred.

Pure FUMUS - the one essential for best results in the flower bed and on the lawn - $\$ 1.00$ per bag; 6 bags for $\$ 5.00$, deliverod. 
Dielytra Spectabilis - Blecaing Eeart. Red flowers; 5 Beet; 4-6th month. Price, $10 \%$ each; \$1.00 per dozen; $\$ 7.50$ per hundred.

Digitalis - Forglove. 3 fect; 7 th month. Price, 10 f each dozen; $\$ 8.00$ per hunared.

Eupatorium Coelestinum - Ageratum. Lavender flowers; 18 inches; 8IIth month.

Fuchsia - Fardy Fuchsia.

F. Americana Elegans. Crimson: 3 feet; 7 -loth month.

F. Nacrostemma. Riccartoni. Cerise-scarlet; 6 feet; 7 -loth month.

Funkia Albo Marzinata - Day Lily; Florers wite and large; 1 to 2 feet; sth montin.

Gailiarda Granaiflcra Vaxima. Blood Crimson, margined with yellow; z feet; 6-loth month.

Geum Atrosanguineam fl. pl. - Barren Strawberry; blood red; 1 to 2 ft; 5-9th month:

Eulalia - Ornamental Grass.

ß. Gracilima Univitatta. Leaves narrow, white rib down center.4-6 ft.

E. Japonica. Green foliage

E. J. Variegata. Leaves stripod; 4 to $6 \mathrm{ft}$.

E. J. Zebrina. Ieaves banded with white; 4-6 ft.

Prices of the above, Iarge clumps, 50 each.

Helenium Autumnale Superbum - Helon Flower. Golden yollow; 5 to 6 ft; 6-loth month.

Helianthus - Perennial Sunflower.

H. Maximilianus. Golden yollow; 5 to $7 \mathrm{ft} ; 9$-loth month.

H. Miss Nellish. Orango Yellow; 6 feet; 8-9th month.

E. IJutiflorus Plenus. Golden; 4 feet; 7 -8th month.

Helleborus Niger - Christmas Rose. Flowers white or tingod with purple; $6-18$ inches; 1-3rd month. Price, $25 d$ each; \$2.50 per dozen.

Eemerocallis - Iellow Day Iily.

H. Apricot. Apricot colored; $2 \frac{1}{2}$ feet; 6 th month.

H. Flava. Canary Yellow; 2 feet; 6 th month.

H. Florham. Golden Yeliov: 3-3 $\frac{1}{2}$ feet; 6-7th month.

H. Gold Dust. Indian Ieliow; $2 \frac{1}{2}$ feet; 5-6th month.

F. Thunbergii. Buttercup Yeliow; 4 feet; 7 th month.

Holiopsis Fitcheriana - crange Sunflower. 2-3 ft; 7-10th month.

Hesperis Viatronalis - Iamo's Violet. Purple, sweet flowers; $2-3 \mathrm{ft}$; 6-8th month.

Heuchera Sanguinea - Alum Root. Bright coral rod flowers; 1 to $1 \frac{1}{2}$ ft; 6-8th month. 
Peonies.

Te have a very larse stock of peonies in thirt-six of the very best lnown verieties - varieties we have tostod out. If interested, ask for our special descriptive list. We can supply them in large clumps that will produce from twonty to twonty-fivo flowers. Young plants, $25 \notin$ each; $\$ 2.50$ per dozon.

Papaver Orientale - Giant Oriental Popjy.

Pentstemon - Beard Tonsue.

P. Barbatus Torreyi. Scarlet red; 3 feet; 7 th month.

P. Digitalis. White; $2-3$ feet; 8 th montr.

P. Gloxinioides "Sensation". Various colors; blooms from early summer to fall. Prices, 10द each; \$1.00 per àozen; \$7.50 per hundred.

Physostegia Virginiana - American Heather. Soft pink; 3-4 fect; 7 to 9th month. Price, 10द each; \$1.00 per dozen; \$7.50 per hundred.

Phlox.

All varieties (at usual rate). Clumps, $25 c^{\prime}$ each; $\$ 2.00$ per dozen; $\$ 15.00$ per hundred. Ask for special list.

Plumbago Ierpentae - Leadwort. Violet blue; 4-6 inches; 6-10th month.

Primula - Hardy Primrose.

P. Elatior. Various colors; 6-9 inches; 4-5th month.

P. Veris superba fl. pl. Yellow; 6 inches; $4-5$ th month. Price, $25 \%$ each; \$2.50 per dozen.

P. Vulgaris. English Primrose. Clumps.

Pyrethrum - Persian Disis; Various colors. 3xtra strong plants 256 each; \$2.50 per dozen; Young plants at usual rate.

Romneya Coulteri - California Tree Poppy. Snowy-thite flowers; 4 ft; 6-10th momth; Price, 506 cach; \$5.00 per dozen.

Rudbeckia - Cono Flower.

R. Golden Glow. Golden Yellow; 5-6 ft; 7-9th month. Price, lof each $\$ 1.00$ per dozen; $\$ 7.50$ per hunared.

R. Naxima. Pure yellow; 5-7 feet; 8-9th month.

Sedum - Stonccrop:

S. Spectabilis. Rose; $1 \frac{1}{2}$ to 2 feet; $9-10 t h$ month.

S. S. Atropurpureum. Rosc; $1 \mathrm{ft;} 9$-loth ronth.

S. S. Brilizint. Red; I foot; 9-10th month.

S. Stoloniferum Coccinoum. Crimson, trailing; 3 inches; 7 -8th month.

Spirea palmata - Goat's Boara. Rosy crimson; 1-2 feet; 6-8th month.

Thalictrum Fendleri - Meaùow Rue. Greonish-white; 1-3 ft; 7-8 month. Price, 25c' each; \$2.50 per dozen

Tradescantia Virginiana - Spider Vort. Violot bluo; 1 to 2 fect;

5-9th month. 
Cempenula - Bell Flower (con.)

C. Medium. White, rose and blue; 5 fest; 6-7ti month.

C. M. Calycahtnema. Wite and rose; 3 feet; $6-7$ th month.

C. Persicifolia Noerheimi. Thite, 2 fect; 6-7th month.

C. Pyramidalis. Blue; 4 to 5 feet; 7 th month.

Callirhoe Inquacratas- Poppy ilallow. Grimson flowers with white centers; 6 inchos; 5 - 8th month.

Caryopteris Mastacanthus - Blue Spirea. Rich violet flowers; 2 feet to 3 feet; 9-10th month.

Herdy chrysanthernums.

Alentown. Larege flowering, yellow

Barney. Iarge yellow, tinged brown.

Boston. Beautiful bronze. Semi-double.

Fairy Quecn. Clear nright pink.

Iva. Fure amoer, fringed petals.

James Boone. Pure white, small flowers.

Iilia. Large terra cotta, very hardy.

Overbrook. Very carly yellow.

Rosinante. Blush rose, dwarf.

Veuve Clicuot. Yellow. bronze, dverf.'

Prices in spring months, potted plants, $12 c^{\prime}$ each; \$1.20 per dozen;

$\$ 10.00$ per hunared. Fielà clumps, $204^{\prime}$ each; \$2.00 per dozen.

Chrysanthenum Maximum - Shasta Daisy.

C. I. Alaska. White; 3 feet; 7 -8th month.

C. II. Shasta Daisy. Pure white; 18 inches; 8-9th month.

Cimicifuga - Snakeroot.

C. Racemosa. Pure white; 3 to 6 feet; 7 -8th month.

C. Simplex. White; 4 to 5 feet; 9-10th month.

Clematis Davidiana - Old Man's Beard. Pale blue; 3 feet; 8-9th inonth.

Convallaria Majalis - Iily of the Valley. Pure white; 6 inches to 1 foot; 3-5th month; Price, 25 each; $\$ 2.50$ per dozen; $\$ 12.00$ per hundred

Coreopsis Ianceolata Grandiflora - Tickseod. Rich golòen yellow; I to 3 feet; 6-9th month. Price, 104 each; 1.00 per dozen; $\$ 7.50$ per hundred.

Dolphinium - Larkspur.

D. Belladonna. Sly blue; 5 to 6 feet; 8 th month.

D. English Hybriàs. BIuo; 6 feet; 7 th month.

D. Chineso. Gontian blue; 2 feet; 7 th month.

D. Elatum. Blue; E to 6 feet; 7 th month.

D. Formosum. Gontian blue:

Fricè, clumps, $25 c^{\prime}$ cach; young plants $15 c^{\prime}$ each.

D. Barbatus (Swoet Villiam)

Dianthus - Eardy Pink.

D. B. Newport Pink. Salmon rose; 18 inches; 6-8th month.

D. Plumarius. 1 foot; 7 -9th month. 
Fibuscus - Mallow Marvels. All colors. Young plants, $25 \%$ each; \$2.50 per dozen. Clumps from field, 50द each; \$5.00 per dozen.

Eypericum lioserianurn - Gold flower. Yellow; 2 ft; 7-8th month.

Eollyhock.

H. Allegheny Fringed. Originated with us, and when introduced the seed brought more than its weight in gold - more than twice as valuable as gold.

H. Newport Fink. Fure pink.

H. Doublo Red, White, Yellow, Pink, Salmon and Maroon. Colors Separate.

Iberis - Candytuft.

I. Gibraltarica. White; 1-2 feet; $3-6$ th month.

I. Sempervirens. Pure wite; $9-12$ inches; $2-8$ th month.

Iris Germanica Pallida Ialmatica - German Iris. Iavender; 40 inches; 6 th month.

Iris Kaempferi - Japanese Iris; 9 varieties. Clumps, 50\& each;

$\$ 5.00$ per dozen.

Iris orientalis Snow Queen. Thite; 3 feet.

Irvandula Vera - Sweet Lavender; 1 to 2 feet; $7-8$ th month. Price, 10 each; \$1.00 per dozen; \$7.50 per hundred.

$$
\text { Lilium - Iillies. }
$$

I. Auratum. Thite; $2-4$ feet; 6 -8th month..

I. Speciosum Album. Pure white; $1-3$ feet; 6-8th month; Price, $20 \%$ each; $\$ 2.00$ per dozen; $\$ 12.00$ per hundred.

L. S. Niagnjficum. Rosy crimson; 1-3 feet; 6-8th month.

L. S. M. Rubra. Pinkish Red; 6-8th month.

I. Candidum. Pure white; 2 to 3 feet; 6 th month.

Iupinus - Iupino.

I. Poluphylius. Jeep bluo; $2-5$ feet; $6-9$ th month.

I. P. Moerhoimi. Pink and white; feet; 6-9th month; Price, $25 \phi^{\prime}$ each; $\$ 2.50$ per dozen.

Irchnis Chalcedonia - Maltese Cross. Scarlet flowers; $2-3$ feet; 6th month; Price, $10 \not$ each: \$1.00 per dozen; \$7. 50 per hundred.

Nionarda Didymoa Rosea - Oswega Tea. Rose; 18 inches; 7-9th month. Price, $10 \zeta^{\prime}$ each; \$1.00 per dozon; \$7.50 per hundred.

IVyosotis Palustris Scmperflorens - Forget-me-not. Blue; 9 inches; 3-8th month; Price, 10\& each; \$1.00 per dozen; \$7.50 per hundrea.

Pachysandra Torminalis - Japanese Spurge. A trailing plant; white; 3 inches; 5 th month. 


\section{SHADE TREES}

ORNAMENTAL. FLOWERING AND

EVERGREEN TREES

SHRUEBERY, ROSEs, VINES.

HARDY PERENNIAL PLANTS.

peonies. grass seeds. etc. FERTILIZERS AND HUMUS.

A. E. WOHLERT, PROPRIETOR
The Garden Nurseries

MONTGOMERY AVENUE'

NARBERTH, PENNA.
GENERAL LANDSCAPE WORK.

PRUNING OF TREES AND

SHRUEBERY.

TELEPHONES

OFFICE. NAREERTH 696

RESIDENCE, NAREERTH 607-D

J. RUSSELL BEBLER, SUPT.

\section{EADY YZRUIIIAIS.

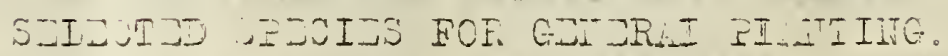

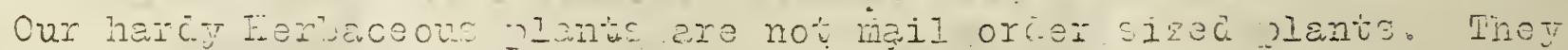

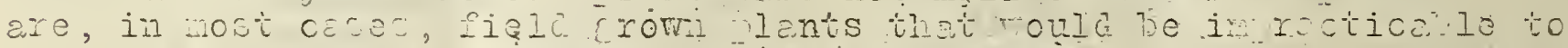
enc by meil.

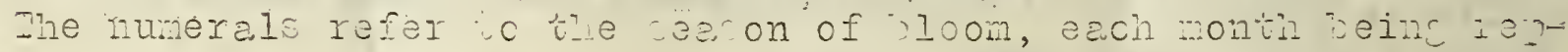

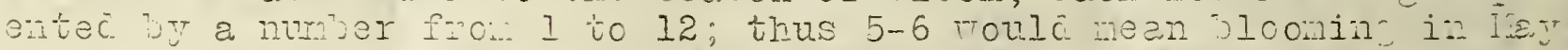
June.

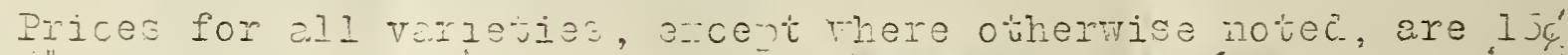

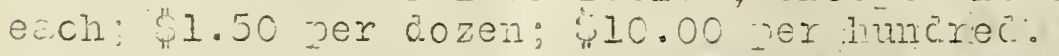

Aconitum Autumale - Ionl-shooc. Ilue; 4 to 5: feet; 9th nonti.

Arostema Coronaria Atrosanvinea - Rose Chamion. Orinson; 1 to 2 feet; 7 th ronth.

Anemone - Tinciflower:

A. Queen Charlotte. Delicaterinl; feet; fall.

1. Roser Suporbe. Jelicate rose; L feet; fall.:

‥ Thirlwind. Snowy whito;. feet: fall

Aruilesia - Columine.

A. Californica Hybrida. Yellow; 50: incher; $5-7$ month:

A. Tenecensis. Scarlet; I to 2 Feet; 4-6thinonth.

A. Chryaintha. Yellow; 5 to 4 feet; 5 -8til month.

4. Coerulea. Blue and hite; 9: to 15 inches: $4-7$ th month.

-. Iivea framiflora. Thite; 12 to 18 inclies; 4-5th wonth.

i. Slimerii. Scarlet and Jellot; y to \& feet: 5-8th"month.

Aster - Michaelmas Dair Ty Peremial Aster.

-. Ame l lus Perry's Favorite ( 1 che? ) Recish Fint; 3 feet.

a. Cranciflorus (5) Derr violet; 5o inches.

$\therefore$ Iovae mliae. (3) Iurple $; 4$ feet.

1. I. A. Eeauty of ColwaII. (2) Iilec blue 4 fest

i. II. $\therefore$. Mrs. J.F. Ramer. (2) Crincon: 4 to 5 feet

$\therefore$ Pertaricum. October; 5 to 6: feet; ros -arijle.

Mie figuras after the varieties incicate their relative tines cf loomin: the all bloom in tre fall)

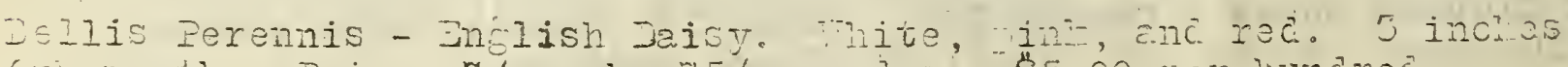

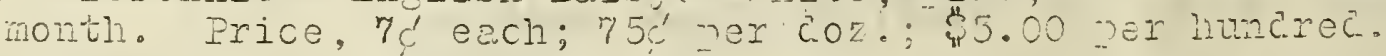

Boltonia - Fense jirrtoit.

Iatis ruma. Pinz to lavencer; to 6 feet; oth month.

I. Tena. Beautiful jint:; 2 feei; etl monti.

\section{Sampanviz - Bell Fiorer.}

corvetica. Porcelain blue; s incies; u- oth month.

víic Impericlis. Verioú colcrs. 